\title{
Análise de correspondência como instrumento para descrição do perfil do trabalhador da cultura de tomate de mesa em Goiás
}

\author{
The correspondence analysis as instrument to analyze the profile of the worker in the tomato \\ crop in the Goiás state, Brazil
}

\author{
Sueli Martins Freitas Alves ${ }^{I^{*}}$ Paulo Marçal Fernandes $^{\mathrm{II}}$ Elton Fialho dos Reis $^{\mathrm{I}}$
}

\section{RESUMO}

O cultivo do tomate de mesa em Goiás está estruturado numa proposta tecnológica baseada no uso intensivo de agrotóxicos, o que envolve riscos e danos potenciais à saúde dos trabalhadores. O objetivo deste trabalho foi utilizar a Análise de Correspondência Múltipla (ACM) para explorar a perfil do trabalhador da cultura de tomate de mesa quanto ao uso de agrotóxicos. Para isso, foram obtidas informações sobre fatores socioeconômicos e demográficos, percepção de risco, uso de agrotóxicos, uso de equipamento de proteção individual (EPI) e morbidade referida, por meio de questionário respondido por 96 trabalhadores da cultura de tomate de mesa, em seis municípios do Estado de Goiás. A Análise de Correspondência Múltipla (ACM) possibilitou definir três grupos de trabalhadores. No grupo 1 , os que responderam que já se intoxicaram com agrotóxicos e não usam EPI porque dificulta o trabalho ou porque não têm costume. No grupo 2, os que responderam que não se intoxicaram com agrotóxico relataram que usam somente algum tipo de EPI porque os consideram desconfortáveis ou por outros motivos. No grupo 3, os que relataram usar EPI completo e que moram em barracas de lona na lavoura de tomate. Foi possível fazer correspondência entre as modalidades da pesquisa, o que não seria possível com o uso de estatística descritiva.

Palavras-chave: exposição aos agrotóxicos, uso de equipamento de proteção individual, análise multivariada

\section{ABSTRACT}

The tomato cropping in the Goiás state is structured under a technological proposal based on the intensive use of pesticides, which involves risks and potential damages to the workers' health. This study aimed to use the Multiple Correspondence Analysis (ACM) to explore the worker's profile concerning to the use of pesticides in tomato crop. So, information about the socioeconomic and demographic factors, risk perception, pesticide uses, use of individual protection equipment (EPI) and referred morbidity were obtained, by using a questionnaire applied to 96 workers in tomato crop, in six counties at the State of Goiás. According to the Multiple Correspondence Analysis, three worker groups were defined. Group 1 involved those workers who answered that they were already intoxicated with pesticides and do not use EPI because it hampers the work or the worker is not accustomed to it. Group 2 referred to those who did not intoxicate with pesticide and use only some EPI type, because they considered them as uncomfortable or other reason. Group 3 were about those who used complete EPI and lived in canvas huts at the farming. It was possible to accomplishing correspondence among the modalities of the research, which would not be possible by using the descriptive statistics.

Key words: exposure to pesticides, using individual-protection equipment, multivariate analysis.

\section{INTRODUÇÃO}

Em 2006, segundo o levantamento do IBGE (2007), o Brasil produziu 3.362.655 toneladas de tomate, sendo os maiores produtores os Estados de Goiás, São Paulo e Minas Gerais, com produções de 759.620t, 672.330t e 552.677t, respectivamente. O Estado de Goiás é composto por 246 municípios agrupados em 18 microrregiões. No ano de 2005, segundo o IBGE, 50 municípios, em oito microrregiões do Estado, produziram tomate de mesa em uma área total estimada de 2.299ha. As microrregiões de Anicuns, Catalão, Anápolis e Entorno do Distrito Federal apresentaram as maiores produtividades de tomate de mesa em 2005, com 54,5t ha-1, 53,3t ha-1, 52,1t ha-1 e 51,7t ha-1, respectivamente (ALVES, 2006).

'Unidade Universitária de Ciências Exatas e Tecnológicas, Universidade Estadual de Goiás (UEG), CP 459, 75110-390, Anápolis, GO, Brasil. E-mail: suelifreitas@ueg.br. *Autor para correspondência.

IIEscola de Agronomia e Engenharia de Alimentos, Universidade Federal de Goiás (UFG), Goiânia, GO, Brasil. 
Ao estudar as causas do uso intensivo de agrotóxicos na cultura de tomate, na região de Goianápolis (GO), REIS FILHO (2002) concluiu que o cultivo está assentado numa proposta tecnológica baseada no uso intensivo de insumos, como adubos e agrotóxicos. O medo de perder a lavoura é muito grande, e o agrotóxico, por ser um produto concreto, visível e que recobre a planta, dá a sensação de segurança para o agricultor. O produtor aprendeu, com o sistema convencional de controle, que a única opção de defesa contra as pragas da cultura é a aplicação desses produtos. Para REIS FILHO (2002), “[...] ficou evidente que as causas do uso de agrotóxicos na cultura têm condicionantes históricos, agronômicos, sociais, comportamentais e econômicos. A tecnologia estabelecida foi se moldando ao longo dos anos ...”.

Vários estudos relatam a importância de observações sobre a relação de exposição química e toxicologia humana. Autilização de agrotóxicos no meio rural brasileiro tem trazido uma série de consequências, tanto para o ambiente, quanto para a saúde do trabalhador rural. Em geral, essas consequências são condicionadas por fatores intrinsecamente relacionados, tais como o uso inadequado desses produtos, a alta toxicidade de certos produtos, a falta de utilização de equipamentos de proteção e a precariedade dos mecanismos de vigilância. Esse quadro é agravado pelo baixo nível socioeconômico e cultural da grande maioria desses trabalhadores (OLIVEIRA-SILVA et al., 2001).

As intoxicações por exposição aos agrotóxicos resultam de uma interação complexa entre as características do agrotóxico e as características da exposição do trabalhador ao produto, incluindo a adoção de medidas e equipamentos de proteção (PERES et al., 2004).

O processo de trabalho agrícola envolve riscos e danos potenciais à saúde dos trabalhadores e deve ser compreendido como a expressão das tecnologias utilizadas, da organização e da divisão do trabalho, da intervenção dos trabalhadores nos locais de trabalho, da ação de técnicos e instituições relacionados à questão e da legislação vigente. É possível afirmar que, nesse processo, além das análises das condições materiais de trabalho, é importante que se atenha também às condições fisiológicas, à experiência acumulada em relação à tarefa e às situações concretas de trabalho nas quais estão inseridos os homens responsáveis pela execução dessas tarefas. Dentre os riscos a que os trabalhadores da cultura de tomate de mesa estão expostos, este trabalho enfoca a intoxicação por agrotóxicos (SILVA et al., 2005).
Este trabalho teve como objetivo utilizar a análise fatorial de correspondência múltipla (ACM) para explorar o perfil do trabalhador da cultura de tomate de mesa, em Goiás, quanto ao uso de agrotóxicos.

\section{MATERIAL E MÉTODOS}

Este trabalho foi realizado em 2005, por meio da aplicação de um questionário aos trabalhadores da cultura de tomate de mesa do Estado de Goiás. Primeiramente, foi obtido um levantamento preliminar dos municípios produtores de tomate de mesa em Goiás. Devido à precariedade de informações sobre o número de lavouras de tomate por município e do número de trabalhadores por propriedade, foi calculado o número de municípios a serem visitados. Com base na área plantada e na produtividade de cada município, a partir das técnicas de amostragem descritas em MEJIA (1973) e COCHRAN (1977), foi estabelecido que o tamanho mínimo da amostra seria de seis municípios. Posteriormente, utilizando-se o processo de amostragem sistemática, foram selecionados os seguintes Municípios: Bonfinópolis, Corumbá de Goiás, Goianápolis, Leopoldo de Bulhões, Pirenópolis e Silvânia.

Antes do início do trabalho de campo, foram adotados dois critérios. O primeiro foi que, em cada município, seriam visitadas somente as lavouras em que a cultura estivesse com mais de 30 dias. $O$ segundo critério foi que seriam entrevistados somente os trabalhadores que estavam envolvidos diretamente com a aplicação de agrotóxicos.

Em cada município selecionado, foi realizado um diagnóstico inicial para o levantamento do número de lavouras que atendiam aos critérios estabelecidos. Além disso, foram realizados, nesta etapa, os contatos com os proprietários dessas lavouras com o objetivo de obter autorização para a realização da pesquisa. Nas lavouras visitadas, foram entrevistados todos os trabalhadores, que lidavam diretamente com os agrotóxicos e, que aceitaram participar da pesquisa.

As questões abordadas consideraram as informações sobre os fatores socioeconômicos e demográficos, tais como a idade, o nível de estudo, o estado civil, a situação funcional, o tempo nesse tipo de serviço e o local onde reside. Também foram levantados os fatores relativos à percepção de risco, ou seja, se os trabalhadores achavam que os agrotóxicos poderiam prejudicar a saúde, se consideravam as informações sobre o uso e se consideravam os cuidados e as recomendações sobre o uso desses produtos. Quanto ao uso de agrotóxicos, foram levantados os produtos usados, a data da última 
aplicação, o intervalo de aplicação, o tempo de duração de cada aplicação, o tipo de pulverizador usado e o tipo de orientação recebida. Em relação ao uso de equipamento de proteção individual (EPI), foram questionados os fatores receptividade ao uso de EPI e motivo para o não uso desses equipamentos. Finalmente, também foi abordada a morbidade referida por meio do levantamento de episódios de intoxicação.

Noventa e seis trabalhadores que lidam com agrotóxicos, na cultura de tomate de mesa em seis municípios produtores do Estado de Goiás, responderam ao questionário (Tabela 1). A tabulação das respostas do questionário gerou uma matriz em que as linhas correspondem aos indivíduos (trabalhadores) e as colunas correspondem às variáveis. As categorias referentes a cada variável, quando necessário, foram reagrupadas e, posteriormente, para que pudesse ser realizada a análise de correspondência múltipla, foram transformadas e codificadas (Tabela 1), conforme MINGOTI (2005), CRIVISQUI (1995) e PEREIRA (1999). Essa tabela, chamada tabela de códigos condensados, não possui propriedades numéricas, pois os códigos representam as categorias de cada variável, portanto não é possível de ser analisada diretamente. É necessário transformá-la em outra que possua propriedades numéricas, mas que resuma o fenômeno observado nos mesmos termos. Por meio de uma recodificação, passa-se à tabela lógica, chamada também tabela disjuntiva completa (TDC). Nessa tabela, as colunas representam as modalidades das variáveis. Na interseção da linha $i$ com a coluna $j$, encontra-se $x i j$, que vale 0 ou 1 , indicando que o indivíduo $i$ possua ou não a modalidade $j$. Um indivíduo está bem representado pelas modalidades que possui, assim, dois indivíduos se assemelham quando apresentam globalmente as mesmas modalidades (MINGOTI, 2005; CRIVISQUI,1995).

A proximidade de dois indivíduos sobre um plano fatorial traduzirá graficamente o fato observado que ambos os indivíduos apresentam um número suficientemente grande de atributos semelhantes nas $p$ variáveis estudadas. Considerando a tabela disjuntiva completa como uma tabela de contingência, a distância usada para avaliar a semelhança entre as linhas (indivíduos) e entre as colunas (modalidades) foi a distância de $\chi^{2}$. A distância entre dois indivíduos cresce na medida em que aumenta o número de modalidades diferentes que esses indivíduos apresentam (VILLAMONTE, 2001; PEREIRA, 1999; CRIVISQUI, 1995).

Um elemento linha ou coluna influi na construção dos eixos por meio de sua inércia em relação ao centro de gravidade (Cont ${ }_{j} \mathrm{aI}_{\mathrm{G}}$ ). Uma variável qualitativa define uma partição sobre o conjunto de indivíduos em tantas classes quanto o número de modalidades. A inércia total (ou variância) de uma variável numérica pode ser descomposta na soma da inércia inter (inércia dos centros de gravidade das classes) e das inércias intra (inércia dos indivíduos em relação ao centro de gravidade da classe que pertencem). A inércia total em relação ao centro de gravidade da nuvem de pontos pode ser obtida pela soma da inércia de cada modalidade (CRIVISQUI, 1995).

A quantidade maximizada pelos eixos fatoriais no espaço $\mathrm{R}_{\mathrm{I}}$ é a inércia projetada da nuvem do conjunto de modalidades. A contribuição de uma variável à inércia de um fator é a soma das contribuições de todas as suas modalidades. A proporção explicada por cada eixo fatorial a em relação à inércia total e dada pela relação entre a taxa de inércia do fator a e a soma da inércia da nuvem de pontos das modalidades projetadas ao longo do eixo fatorial a (MINGOTI, 2005; PEREIRA 1999; CRIVISQUI, 1995).

A etapa seguinte do trabalho consiste em agrupar as unidades utilizando o método da classificação hierárquica ascendente. Retornando à nuvem de pontos que representa os trabalhadores (unidades), na qual, as dimensões do espaço são dadas pelas variáveis, pode-se agrupar esses pontos segundo os grupamentos naturalmente formados, em função dos diferentes desempenhos das unidades. É exatamente por terem perfil semelhante que os trabalhadores se localizam em pontos relativamente próximos no espaço. O critério de agrupamento de pontos utilizado neste trabalho foi o método de Ward, por ser o método no qual os grupos resultantes representam a menor redução da variação total da matriz. A descrição qualitativa das classes é feita por meio das categorias com maior significado na construção de cada classe (LEBART et al., 1984).

O pacote computacional estatístico utilizado foi o Sistema Portátil de Análise de Dados Numéricos $\left(\mathrm{SPADN}^{\circledR}\right)$. Para realizar a análise, foram utilizadas 12 variáveis com um total de 37 modalidades, e estas foram divididas em ativas e suplementares ou ilustrativas. As variáveis: cuidados e recomendações, uso de EPI, motivo do não uso de EPI, orientação técnica e sintomas de intoxicação, foram consideradas como variáveisativas e entraram no cálculo dos fatores gerados pela análise. As demais variáveis foram consideradas ilustrativas, de peso nulo, e foram plotadas no mapa sem interferir no cálculo dos eixos fatoriais.

Ciência Rural, v.39, n.7, out, 2009. 
Tabela 1 - Descrição e distribuição das características socioeconômicas (n), percepção de risco, uso de agrotóxicos e uso de EPI, apresentados no questionário aplicado aos trabalhadores da cultura de tomate de mesa em seis municípios do Estado de Goiás, 2005.

\begin{tabular}{|c|c|c|c|c|c|c|}
\hline Fatores & Variável & Categorias & $\begin{array}{c}\text { Categoria } \\
\text { transformada }\end{array}$ & Codificação & $\mathrm{n}$ & $\%$ \\
\hline \multirow{9}{*}{ 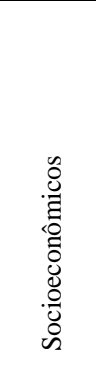 } & \multirow{4}{*}{ Grau de instrução } & Sem estudo & 1 & GI01 & 4 & 4,2 \\
\hline & & Ensino Fundamental incompleto & 2 & GI02 & 74 & 77,1 \\
\hline & & Ensino Fundamental completo & 3 & GI03 & 12 & 12,5 \\
\hline & & Ensino Médio & 4 & GI04 & 6 & 6,3 \\
\hline & \multirow{5}{*}{$\begin{array}{l}\text { Tempo de Serviço } \\
\text { neste tipo de } \\
\text { atividade }\end{array}$} & $<5$ & 1 & TES1 & 8 & 8,3 \\
\hline & & 05 |--- 10 & 2 & TES2 & 14 & 14,6 \\
\hline & & $10 \mid--15$ & 3 & TES3 & 18 & 18,8 \\
\hline & & $15 \mid---20$ & 4 & TES4 & 30 & 31,3 \\
\hline & & $=20$ & 5 & TES5 & 26 & 27,1 \\
\hline \multirow{8}{*}{ 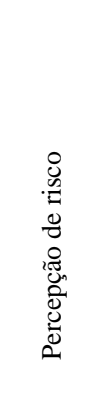 } & \multirow{2}{*}{ Local onde reside } & Casa na cidade & 1 & RES1 & 64 & 66,7 \\
\hline & & Barraca de lona na lavoura & 2 & RES2 & 32 & 33,3 \\
\hline & \multirow{3}{*}{$\begin{array}{l}\text { Informação sobre } \\
\text { perigo }\end{array}$} & Não sabe & 1 & INF1 & 20 & 20,8 \\
\hline & & Acredita & 2 & INF2 & 70 & 72,9 \\
\hline & & Não acredita & 3 & INF3 & 6 & 6,3 \\
\hline & \multirow{3}{*}{$\begin{array}{l}\text { Cuidados e } \\
\text { recomendações }\end{array}$} & Não sabe & 1 & REC1 & 6 & 6,3 \\
\hline & & Segue & 2 & REC2 & 38 & 39,6 \\
\hline & & Não segue & 3 & REC3 & 52 & 54,2 \\
\hline \multirow{8}{*}{ 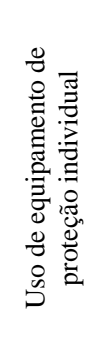 } & \multirow{8}{*}{ Uso de EPI } & Não usa & 1 & EPI1 & 48 & 50,0 \\
\hline & & Usa incompleto & 2 & EPI2 & 28 & 29,2 \\
\hline & & Usa completo & 3 & EPI3 & 20 & 20,8 \\
\hline & & Dificulta o trabalho & 1 & JUS1 & 10 & 10,4 \\
\hline & & Desconfortável & 2 & JUS2 & 26 & 27,1 \\
\hline & & Falta de costume & 3 & JUS3 & 30 & 31,3 \\
\hline & & Outro & 4 & JUS4 & 10 & 10,4 \\
\hline & & Usa EPI & 5 & JUS5 & 20 & 20,8 \\
\hline \multirow{9}{*}{ 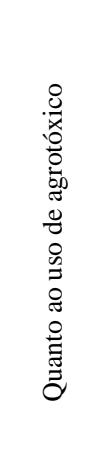 } & \multirow{2}{*}{ Tipo de pulverizador } & Costal & 1 & TIP1 & 48 & 50,0 \\
\hline & & Motorizado & 2 & TIP2 & 48 & 50,0 \\
\hline & \multirow{2}{*}{$\begin{array}{l}\text { Tempo de } \\
\text { exposição(h) }\end{array}$} & Até duas horas & 1 & APL1 & 80 & 83,3 \\
\hline & & Mais de duas horas & 2 & APL2 & 16 & 16,7 \\
\hline & \multirow{3}{*}{$\begin{array}{l}\text { Intervalo de } \\
\text { pulverização (dias) }\end{array}$} & Não tem dia definido & 1 & PUL1 & 18 & 18,8 \\
\hline & & Dois dias & 2 & PUL2 & 34 & 35,4 \\
\hline & & Três dias & 3 & PUL3 & 44 & 45,8 \\
\hline & \multirow{2}{*}{ Quanto à utilização } & Não recebe orientação técnica & 1 & ORT1 & 26 & 27,1 \\
\hline & & Recebe orientação técnica & 2 & ORT2 & 70 & 72,9 \\
\hline \multirow{2}{*}{ 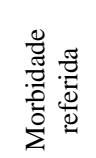 } & \multirow[b]{2}{*}{ Relato de sintomas } & Não relatou sintomas de intoxicação & 1 & INT1 & 70 & 72,9 \\
\hline & & Relatou sintomas de intoxicação & 2 & INT2 & 26 & 27,1 \\
\hline
\end{tabular}

Ciência Rural, v.39, n.7, out, 2009. 


\section{RESULTADOS E DISCUSSÃO}

O tomateiro é cultivado no Brasil durante o ano todo, e a variação do preço e da produtividade está diretamente relacionada à ocorrência de insetospraga e de doenças que causam grandes perdas e oneram o custo da produção. Devido ao ataque de pragas à cultura, os tomaticultores lançam mão de medidas de controle que são utilizadas de forma não planejada, por meio do sistema convencional, ou seja, o método químico. Dentre os inseticidas comerciais mais usados nas lavouras visitadas, estão: Tamaron (organofosforado), Cefanol (organofosforado), Vertimec (avermectina), Furandan 50G (carbamato), Mospilan (neonicotinóide), Confidor (neonicotinóide), Decis (piretróide). A promoção de estratégias baseadas na necessidade real do uso de agrotóxicos apresenta alguns problemas, na medida em que os agricultores se mostram relutantes na redução do uso desses produtos, pois temem colocar sob risco os investimentos feitos em suas culturas (GARCIA, 2001). Os produtores de tomate de mesa em Goiás, para garantirem a produção e o controle das pragas que atacam a cultura, continuam se apoiando em pacotes tecnológicos, utilizando a aplicação sistemática de produtos químicos. Esse fato propicia aos trabalhadores uma exposição constante aos agrotóxicos (ALVES, 2006).

Para analisar os dados que descrevem as respostas dos trabalhadores que lidam com agrotóxicos, na cultura de tomate, em seis municípios do Estado de Goiás, os dados foram resumidos (Tabela 1) e foi realizada a Análise de Correspondência Múltipla (ACM). Dentre os objetivos da Análise de Correspondência Múltipla (ACM), estão explorar as relações que podem existir entre os indivíduos e/ou entre eles e as características avaliadas, representar graficamente a estrutura da tabela e facilitar a construção de tipologias de indivíduos. Para que se possa efetuar a comparação desejada, é necessário que se faça uma representação gráfica das $i$ unidades de observação no espaço das k modalidades e vice-versa. Nesse sentido, o propósito da análise fatorial é reduzir a dimensionalidade do sistema, a partir do emprego da métrica de chi-quadrado $(\chi 2)$. Como resultado final, pode-se estabelecer grupos com características semelhantes, explorando assim o perfil dos trabalhadores da cultura de tomate (CRIVISQUI,1995; LEBART et al., 1984).

Para gerar a ACM, foram consideradas cinco variáveis ativas com um total de 15 modalidades e sete suplementares com 22 modalidades, mas estas últimas não foram usadas para gerar a análise. A primeira etapa na interpretação da ACM consistiu em definir o número de fatores a serem selecionados para compor os planos fatoriais, e essa decisão se baseou na análise da decomposição da inércia total da população estudada. Verifica-se que os dois primeiros eixos retêm 38,88\% da variabilidade total. Não há regras estabelecidas definindo até que plano os gráficos devem ser analisados. Neste trabalho, será apresentado o gráfico composto pelos dois primeiros eixos. O eixo 1 retém 20,11\% da variabilidade dos dados, e o eixo 2 retém $18,77 \%$. A partir da determinação do número de eixos fatoriais, pode-se fazer o gráfico de dispersão das coordenadas das modalidades, conhecido como gráfico de correspondência (Figura 1).

No primeiro plano fatorial, estão projetadas todas as variáveis ativas analisadas (Figura1a). Contudo, elas contribuem diferentemente na geração da inércia projetada nele. A tabela 2 apresenta a distância total de cada modalidade ao centro de gravidade, suas coordenadas e contribuições e a contribuição de cada variável na geração da inércia dos dois primeiros eixos. A soma das contribuições em cada eixo deve igual a $100 \%$, sendo assim, verifica-se que as variáveis que se referem ao uso de equipamento de proteção individual (EPI) e ao motivo de não uso desses equipamentos (JUS) foram as que mais contribuíram para geração dos eixos, com $42,50 \%$ e $41,00 \%$, respectivamente, no eixo 1, e com 42,60\% e 35,70\%, no eixo 2.

Para orientar a análise das variáveis suplementares (Figura 1b), pode ser usada a distância de cada ponto à origem do eixo, em número de desviospadrão, conhecida por valor teste. Utilizando a probabilidade de $5 \%$ de ocorrência casual da localização de determinado ponto no eixo, são considerados significativos os pontos com valor teste acima de dois, em números absolutos (CRIVISQUI, 1995). Assim, as modalidades ilustrativas que apresentam associação significativa com o eixo 1 são: reside em casa na cidade (RES1), reside em barraca de lona na lavoura (RES2), não sabe sobre o perigo do uso de agrotóxicos (INF1), gasto de até duas horas para pulverização dos agrotóxicos na lavoura (APL1), gasto de mais de duas horas para pulverização dos agrotóxicos na lavoura (APL2), o intervalo de aplicação de agrotóxicos é em média de dois dias (PUL1) e o intervalo é em média de três dias (PUL2). Com o eixo 2, são o tempo de serviço nesse tipo de atividade é de mais de 20 anos (TES5), reside em casa na cidade (RES1), reside em barraca de lona na lavoura (RES2), não sabe sobre o perigo do uso de agrotóxicos (INF1), acredita nas informações sobre o perigo do uso de agrotóxicos (INF2), não tem dia definido para aplicação de agrotóxicos (PUL1) e o intervalo para aplicação é em média de dois dias (PUL2).

Após a ACM, fez-se a análise de agrupamento pelo método da classificação hierárquica ascendente, o que possibilitou definir três tipos de grupos de trabalhadores (Figura 1 e Tabela 3). 


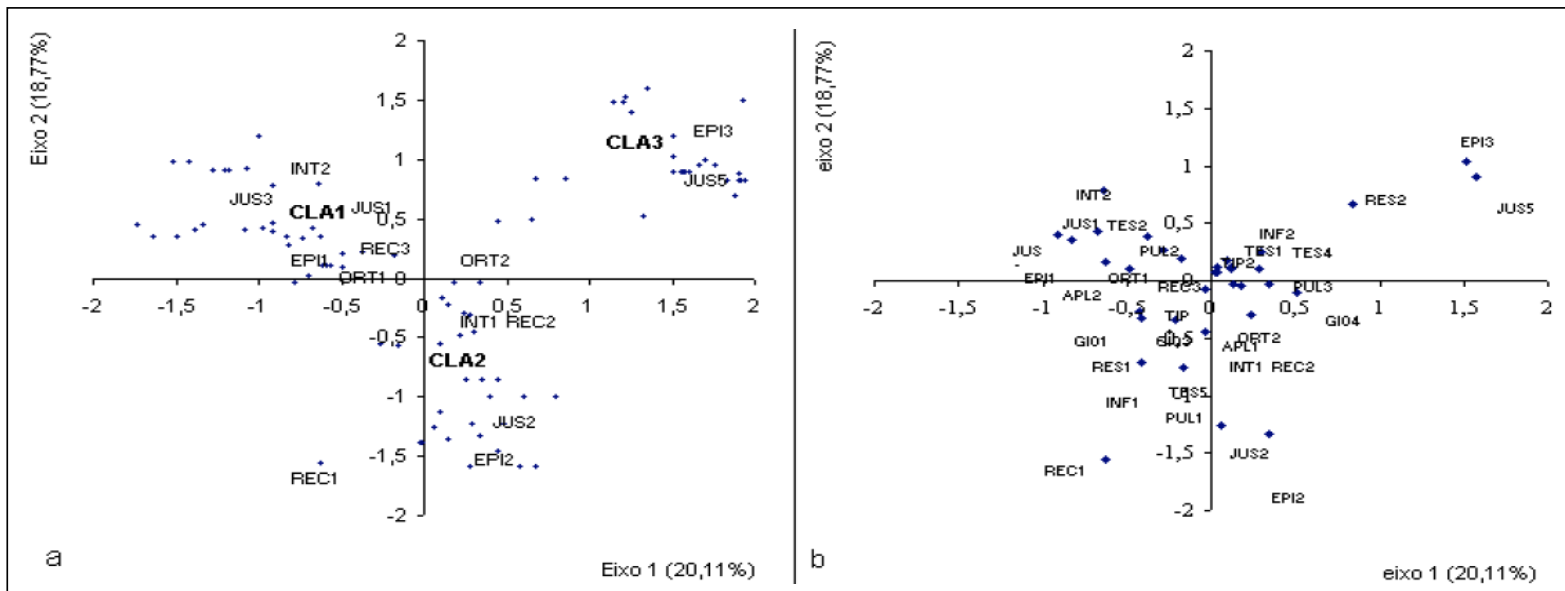

Figura 1 - Análise de correspondência múltipla para os indivíduos e as variáveis ativas (a): cuidados e recomendações (REC1 = não sabe; REC2 = segue; REC3 = não segue), uso de EPI (EPI1 = não usa; EPI2 = usa incompleto; EPI3 = usa completo), motivo do não uso de EPI (JUS1 = dificulta o trabalho, JUS2 = desconfortável, JUS3 = falta de costume, JUS4 = outros motivos, JUS5 = usa EPI), orientação técnica (ORT1 = não recebe orientação técnica, ORT2 = recebe orientação técnica) e relato de sintomas de intoxicação (INT1 = não relatou, INT2 = relatou), e ativas e suplementares (b): grau de instrução (GI01 = sem estudo, GI02 = Ensino Fundamental incompleto, GI03 = Ensino Fundamental completo, GI04 = Ensino Médio), tempo neste tipo de atividade (TES1 $=<5$, TES2 $=05 \mid-10$, TES3 $=10 \mid-15$, TES4 = $15 \mid-20$, TES5 $\geq 20$ ), local onde reside (RES1 = casa na cidade, RES2 = barraca de lona na lavoura), se acredita na informação sobre perigo do uso de agrotóxicos (INF1 = não sabe, INF2 = acredita, INF3 = não acredita), tipo de pulverizador (TIP1 = costal, TIP2 = motorizado), tempo de exposição (APL1 = até duas horas, APL2 = mais de duas horas), intervalo de pulverização (PUL1 = não tem dia definido, PUL2 = em média a cada dois dias, PUL3 = em média a cada três dias). CLA1=classe 1 ; CLA2 = classe 2 ; CLA3 = classe 3.

Tabela 2 - Distância de cada modalidade ao centro de gravidade (dist), coordenadas e contribuição (\%) da modalidade na geração da inércia e das variáveis ativas, nos dois primeiros eixos fatoriais.

\begin{tabular}{|c|c|c|c|c|c|c|c|c|c|}
\hline \multirow{2}{*}{ Variáveis ativas } & \multirow{2}{*}{ Categorias } & \multirow{2}{*}{ Cód. } & \multirow{2}{*}{ dist } & \multicolumn{2}{|c|}{ Coordenadas } & \multicolumn{2}{|c|}{ Contribuição } & \multicolumn{2}{|c|}{ Valor teste } \\
\hline & & & & Eixo1 & Eixo2 & Eixo1 & Eixo2 & Eixo1 & Eixo2 \\
\hline \multirow{4}{*}{$\begin{array}{l}\text { Cuidados e } \\
\text { Recomendações }\end{array}$} & Não sabe & REC1 & 15,00 & $-0,63$ & $-1,55$ & 1,20 & 8,00 & $-1,6$ & $-3,9$ \\
\hline & Segue & REC2 & 1,53 & 0,34 & $-0,03$ & 2,30 & 0,00 & 2,7 & $-0,3$ \\
\hline & Não segue & REC3 & 0,85 & $-0,18$ & 0,20 & 0,80 & 1,20 & $-1,9$ & 2,2 \\
\hline & Contribuição acumulada & & & & & 4,40 & 4,40 & & \\
\hline \multirow{4}{*}{ Uso de EPI } & Não usa & EPI1 & 1,00 & $-0,83$ & 0,35 & 17,10 & 3,30 & $-8,1$ & 3,4 \\
\hline & Usa incompleto & EPI2 & 2,43 & 0,34 & $-1,33$ & 1,70 & 27,70 & 2,1 & $-8,3$ \\
\hline & Usa completo & EPI3 & 3,80 & 1,51 & 1,03 & 23,7 & 11,70 & 7,6 & 5,1 \\
\hline & Contribuição acumulada & & & & & 42,50 & 42,50 & & \\
\hline \multirow{6}{*}{$\begin{array}{l}\text { Motivo do não uso } \\
\text { EPI }\end{array}$} & Dificulta o trabalho & JUS1 & 8,60 & $-0,68$ & 0,43 & 2,40 & 1,00 & $-2,3$ & 1,4 \\
\hline & Desconfortável & JUS2 & 2,69 & 0,06 & $-1,26$ & 0,10 & 22,90 & 0,4 & $-7,5$ \\
\hline & Falta de costume & JUS3 & 2,20 & $-0,91$ & 0,40 & 12,90 & 2,70 & $-6,0$ & 2,7 \\
\hline & Outro & JUS4 & 8,60 & 0,11 & $-0,16$ & 0,10 & 0,10 & 0,4 & $-0,5$ \\
\hline & Usa EPI & JUS5 & 3,80 & 1,57 & 0,90 & 25,60 & 9,00 & 7,9 & 4,5 \\
\hline & Contribuição acumulada & & & & & 41,00 & 41,00 & & \\
\hline \multirow{3}{*}{$\begin{array}{l}\text { Orientação sobre o } \\
\text { uso de agrotóxicos }\end{array}$} & Não recebe orientação técnica & ORT1 & 2,69 & $-0,49$ & 0,10 & 3,30 & 0,20 & $-2,9$ & 0,6 \\
\hline & Recebe orientação técnica & ORT2 & 0,37 & 0,18 & $-0,04$ & 1,20 & 0,10 & 2,9 & $-0,6$ \\
\hline & Contribuição acumulada & & & & & 4,50 & 4,50 & & \\
\hline \multirow{3}{*}{ Morbidade referida } & Não relatou sintomas de intoxicação & INT1 & 0,37 & 0,24 & $-0,29$ & 2,10 & 3,30 & 3,8 & $-4,7$ \\
\hline & Relatou sintomas de intoxicação & INT2 & 2,69 & $-0,64$ & 0,79 & 5,60 & 8,90 & $-3,8$ & 4,7 \\
\hline & Contribuição acumulada & & & & & 7,60 & 7,60 & & \\
\hline
\end{tabular}


Tabela 3 - Distribuição dos trabalhadores por classe, conforme análise de agrupamento hierárquico.

\begin{tabular}{|c|c|c|c|c|c|}
\hline \multicolumn{2}{|c|}{ Distribuição por classe } & Classe 1 & Classe 2 & Classe 3 & Total \\
\hline \multicolumn{2}{|c|}{ Número de trabalhadores por classe } & 40 & 34 & 22 & 96 \\
\hline \multicolumn{2}{|c|}{$\%$ de trabalhadores por classe } & 41,67 & 35,42 & 22,91 & 100 \\
\hline \multirow{6}{*}{ 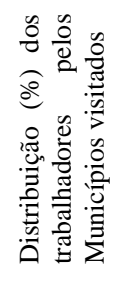 } & Goianápolis & 35,0 & 58,8 & 0 & 35,4 \\
\hline & Leopoldo de Bulhões & 10,0 & 5,9 & 0 & 6,3 \\
\hline & Bonfinópolis & 25,0 & 11,8 & 0 & 14,8 \\
\hline & Silvânia & 15,0 & 11,8 & 0 & 10,4 \\
\hline & Corumbá de Goiás & 0 & 0 & 54,5 & 12,5 \\
\hline & Pirenópolis & 15,0 & 11,8 & 45,5 & 20,8 \\
\hline \multicolumn{2}{|c|}{$\begin{array}{l}\text { Características significativas* associadas a cada } \\
\text { classe }\end{array}$} & $\begin{array}{l}\text { EPI1; JUS1; JUS3 } \\
\text { INT2; RES1. }\end{array}$ & $\begin{array}{l}\text { EPI2; JUS2; JUS4 } \\
\text { INT1; RES1. }\end{array}$ & EPI3; JUS5; RES2. & - \\
\hline
\end{tabular}

*: características que apresentaram o valor teste significativo (> 2,0) em relação à classe avaliada. As demais modalidades apresentaram o valor teste inferior a 2,0. Uso de EPI (EPI1 = não usa; EPI2 = usa incompleto; EPI3 = usa completo), motivo do não uso de EPI (JUS1 = dificulta o trabalho, JUS2 = desconfortável, JUS3 = falta de costume, JUS4 = outros motivos, JUS5 = usa EPI), relato de sintomas de intoxicação (INT1 = não relatou, INT2 = relatou), local onde reside (RES1 = casa na cidade, RES2 = barraca de lona na lavoura).

a) No 2o quadrante, tem-se o grupo 1 , com $41,67 \%$ dos trabalhadores que estão associados às seguintes características significativas: os trabalhadores responderam que já se intoxicaram com agrotóxicos (INT2), não usam equipamento de proteção individual (EPI1) porque dificulta o trabalho (JUS1) ou porque não têm costume (JUS3);

b) No $4^{\circ}$ quadrante, tem-se o grupo 2, com $35,42 \%$ dos trabalhadores que estão associados às seguintes características significativas: os trabalhadores responderam que não se intoxicaram com agrotóxico (INT1), usam somente algum tipo de equipamento de proteção individual (EPI2) porque os consideram desconfortáveis (JUS2) ou por outros motivos (JUS4);

c) No $1^{\circ}$ quadrante, tem-se o grupo 3 , com $22,91 \%$ dos trabalhadores que estão associados às seguintes características significativas: os trabalhadores que disseram usar equipamento de proteção individual (EPI3) e moram em barracas de lona, na lavoura.

Alguns aspectos merecem ser destacados, tais como o fato de que nesta análise não foram consideradas as condições de trabalho e nem as condições de uso dos equipamentos de proteção individual (EPI).

\section{CONCLUSÕES}

As variáveis que apresentaram maior contribuição para descrever o perfil do trabalhador da cultura de tomate de mesa, foram o uso de equipamento de proteção, relato de sintomas de intoxicação e motivo para o não uso de equipamento de proteção. Com base na análise de correspondência, identificaram-se três tipos distintos de trabalhadores quanto ao uso de equipamento de proteção individual. As variáveis cuidados e recomendações e assistência técnica não apresentaram associação significativa com um grupo específico de trabalhadores. Esse fato se deve provavelmente à carência de assistência técnica e a não inserção de um conjunto de recomendações e cuidados que devem ser observados no uso de agrotóxicos, e que devem fazer parte da rotina de trabalho desses trabalhadores.

\section{REFERÊNCIAS}

ALVES, S.M.F. Condições de trabalho e percepção de riscos associados ao uso de agrotóxicos na cultura de tomate de mesa em Goiás. 2006. 93f. Tese (Doutorado em Agronomia: Produção Vegetal) - Escola de Agronomia e Engenharia de Alimentos, Universidade Federal de Goiás, Goiânia, GO.

COCHRAN, W.G. Sampling techiques. 3.ed. New York: John Wiley \& Sons. 1977. 555p.

CRIVISQUi, E. Apresentação da análise fatorial de correspondência simples e múltiplas. Programme de Recherche et D’Enseignement en Statistique Appliquée. PRESTA, Belgique: Université Libre de Bruxelles, 1995. 162p. (Apostila).

GARCIA, E.G. Segurança e saúde no trabalho rural: a questão dos agrotóxicos. São Paulo: Fundacentro, 2001. 182p.

Ciência Rural, v.39, n.7, out, 2009. 
INSTITUTO BRASILEIRO DE GEOGRAFIA E ESTATÍSTICA (IBGE). Levantamento sistemático da produção agrícola, ano 2006. Disponível em http://www.ibge.gov.br/home/ estatistica/indicadores/agropecuaria. Acesso em 13 de março de 2007.

LEBART, L. et al. Multivariate descriptive statistical analysis: correspondence analysis and related techniques. New York: John Wiley \& Sons, 1984. 250p.

MEJIA, R. Procedimentos para estudios de prevalencia de enfermedades cronicas en el ganado. Buenos Aires: Centro Panemericano de Zoonosis, 1973. 33p. (Nota técnica n. 18).

MINGOTI, S.A. Análise de dados através de métodos de estatística multivariada: uma abordagem aplicada. Belo Horizonte: UFMG, 2005. 297p.

OLIVEIRA-SILVA, J.J. et al. Influência de fatores socioeconômicos na contaminação por agrotóxicos, Brasil. Revista de Saúde Pública, São Paulo, v.35, n.2, p. 130-135. 2001. Disponível em: $<$ http://www.scielo.br/scielo.php?script=sci_arttext\&pid=S003489102001000200005\&lng=pt\&nrm=iso $>$. doi: 10.1590/S003489102001000200005.

PEREIRA JCR. Análise de dados qualitativos: estratégias metodológicas para as ciências da saúde, humanas e sociais. São Paulo: EDUSP, 1999. 157p.
PERES, F. et al. Percepção das condições de trabalho em uma tradicional comunidade agrícola em Boa Esperança, Nova Friburgo, Rio de Janeiro, Brasil. Cadernos de Saúde Pública. Rio de Janeiro, v.20, n.4, p.1559-1568. 2004. Disponível em: $<$ http://www.scielo.br/scielo.php?script=sci_arttext\&pid=S0102311X2004000400021\&lng=pt\&nrm=iso>. doi: 10.1590/S0102$311 X 2004000400021$.

REIS FILHO, J.S. Agrotóxicos na cultura do tomateiro (Lycopersicon esclentum): Causas do uso intensivo. 2002. 140f. Dissertação (Mestrado em Agronomia) - Escola de Agronomia, Universidade Federal de Goiás, Goiânia, GO.

SILVA, J.M. et al. Agrotóxico e trabalho: uma combinação perigosa para a saúde do trabalhador rural. Ciência e Saúde Coletiva. Rio de Janeiro, v.10, n.4, p. 891-903. 2005. Disponível em: $<$ http://www.scielo.br/scielo.php?script=sci_arttext\&pid=S141381232005000400013\&lng=pt\&nrm=iso $>$. ISSN 1413-8123. doi: $10.1590 / \mathrm{S} 1413-81232005000400013$.

SISTEMA PORTATÍL DE ANÁLISE DE DADOS NUMÉRICOS (SPADN). Centre international de statistique ET d'informatique appliqués (CISIA). Version 2.5. France: Saint-Mandé, 1993. 215p.

VILLAMONTE, G. Proposta metodológica para construção de indicadores para avaliação da rede escolar brasileira. 2001. 170f. Dissertação (Mestrado em Engenharia de Produção) - Programa de Pós-graduação em Engenharia de Produção, UFSC, Florianópolis, SC. 